\title{
Modelo matemático para estimar el índice de calidad de vida en adultos mayores usando la encuesta FUMAT
}

\author{
Johana Vanessa Meza Paredes (iD), Ariel Insaurralde Alviso (iD), \\ Dario Alviso (iD) 1 \\ ${ }^{1}$ Universidad María Auxiliadora. Mariano Roque Alonso, Paraguay \\ ${ }^{2}$ Universidad Nacional de Asunción, Instituto de Investigaciones en Ciencias de la \\ Salud, San Lorenzo, Paraguay
}

Cómo referenciar este artículo/ How to reference this article:

\begin{abstract}
Meza Paredes JV, Insaurralde Alviso A, Alviso D. Modelo matemático para estimar el índice de calidad de vida en adultos mayores usando la encuesta FUMAT. Mem. Inst. Investig. Cienc. Salud. 2021; 19(3): 44-53
\end{abstract}

\begin{abstract}
RES U M E N
Son muchas las personas en el mundo actual que dedican su esfuerzo a lo que podría denominarse "la nueva cultura de la longevidad", que es el intento de vivir más y en mejores condiciones de vida. El objetivo de este trabajo es proponer un modelo matemático para estimar el índice de calidad de vida (ICV) en adultos mayores. El caso de estudio corresponde a ancianatos de la zona periurbana de Asunción, Paraguay. El estudio es observacional descriptivo de corte transversal. Participaron 33 adultos mayores de 60 años, de ambos sexos, para los cuales se estimó el ICV a través de la encuesta Evaluación del ICV en adultos mayores (FUMAT). Se aplicó una regresión múltiple lineal para estimar el ICV a través de un modelo matemático a partir de los parámetros de distintos indicadores. Respecto a los indicadores de la calidad de vida, se encontró que los promedios más bajos de los distintos indicadores corresponden a los relacionados al derecho (43 \pm 19$)$, el bienestar material $(45 \pm 30)$ y la autodeterminación $(48 \pm 24)$. El modelo propuesto presenta un excelente acuerdo con los datos iniciales. Los valores de error estándar y RMSE son $0.22 \%$ y 0.10 , respectivamente. Además, el modelo reproduce correctamente la dependencia de los distintos indicadores con el valor del ICV. Asimismo, permite identificar cuáles son los indicadores con más peso en la estimación del ICV, y permite predecir su valor en otras muestras.
\end{abstract}

Palabras clave: Adulto mayor, Calidad de vida, Regresión, Modelo matemático.

\section{Mathematical model to estimate the quality of life index in older adults using the FUMAT survey}

\begin{abstract}
A B S T R A C T
Many people nowadays dedicate their efforts to "the new culture of longevity", which is an attempt to live longer and in the best conditions of life. The objective is to propose a mathematical model to estimate the quality of life index (ICV) in older adults. The case study corresponds to nursing homes in the periurban area of Asunción, Paraguay. This is a cross-sectional descriptive observational study. 33 adults over 60 years of age, of both sexes, participated, for whom the ICV was estimated through the ICV Assessment in Older Adults (FUMAT) survey. A multiple linear regression analysis was conducted to estimate the ICV using parameters of different indicators. Regarding the quality of life indicators, it was found that the
\end{abstract}

Fecha de recepción: octubre 2021. Fecha de aceptación: noviembre 2021

*Autor correspondiente: Dario Alviso. Universidad María Auxiliadora. Mariano Roque Alonso, Paraguay

Tel: (549) 1133223320

Email: beto.alviso@gmail.com 
lowest averages concerning the indicators correspond to those related to law ( $43 \pm$ $19)$, material well-being $(45 \pm 30)$ and self-determination $(48 \pm 24)$. The proposed model presents an excellent agreement with the initial data. The standard error and RMSE values are $0.22 \%$ and 0.10 , respectively. Furthermore, the model correctly reproduces the dependence of the different indicators on the ICV value. In addition, it allows us to identify the indicators with the most weight in estimating the ICV, and to predict its value in other samples.

Keywords: Older adult, Quality of life, Regression, Mathematical model.

\section{INTRODUCCIÓN}

En la actualidad muchas personas dedican sus esfuerzos a lo que se denomina plena calidad de vida en el envejecimiento, que consiste en llegar a ser un adulto mayor con buenas condiciones físicas, mentales y sociales, de modo a enriquecer todas las dimensiones que involucran la calidad de vida de los adultos mayores ${ }^{(1-3)}$. La calidad de vida es el estado de bienestar físico, social, emocional, espiritual, intelectual y ocupacional que le permite al individuo satisfacer apropiadamente sus necesidades individuales, colectivas y por ende mantener un buen estado de salud ${ }^{(4-5)}$. Existen varios factores asociados a la calidad de vida de los adultos mayores ${ }^{(6-7)}$. Además, su estudio en hogares de ancianos o geriátricos ha sido objeto de investigación en los últimos años ${ }^{(8-9)}$.

Tradicionalmente la determinación del estado de salud o enfermedad de los individuos se ha centrado en métodos objetivos basados en la elaboración e implementación de mediciones de parámetros, tales como marcadores bioquímicos, fisiológicos y anatómicos, o simplemente empleando el uso de indicadores epidemiológicos para caracterizar una población. Sin embargo, existía un vacío instrumental para medir la calidad de vida o estado de salud individual en función de la definición de salud planteada por la Organización Mundial de la Salud. Su medición no ha sido sencilla, pero varias investigaciones han logrado definir instrumentos para evaluarla(10-11).

En la presente investigación se ha utilizado la Escala FUMAT, Escala Integral de la Calidad de Vida. Esta escala de Calidad de Vida es aplicable a personas mayores como una estrategia para evaluar y mejorar la calidad de sus servicios, programas y apoyos, y está adaptada a este grupo de población y a sus necesidades y realidad. La importancia del instrumento radica principalmente en que no existen en nuestro contexto muchos instrumentos adecuados para medir la calidad de vida en una población. La calidad de vida es evaluada a través de 57 ítems distribuidos en ocho subescalas: Bienestar emocional, Relaciones interpersonales, Bienestar material, Desarrollo personal, Bienestar físico, Autodeterminación, Inclusión social y Derechos. Según se vayan seleccionando los ítems son asignadas calificaciones que dan lugar a las puntuaciones por dimensión y luego haciendo una sumatoria de las dimensiones se obtendrá el diagnóstico del Índice de Calidad de Vida (ICV) ${ }^{(12-13)}$.

Por otro lado, la estadística inferencial permite, entre otras cosas, proponer modelos matemáticos o correlaciones, a partir de una base de datos, que le permiten obtener tendencias para todos los datos numéricos, así como para predecir estos valores para otras condiciones iniciales ${ }^{(14)}$. Los modelos de regresión permiten encontrar una correlación entre las diferentes variables de entrada y salida. Por esta razón, hay varias técnicas de regresión para problemas lineales y no lineales.

Para problemas de correlación lineal, la regresión múltiple lineal utiliza el método de los mínimos cuadrados para encontrar los coeficientes de los diferentes términos lineales de la ecuación, de forma tal a que los valores estimados por la ecuación sean los más cercanos posible a los valores de la variable de salida ${ }^{(15)}$.

Dado que el método tradicional para la estimación del ICV exige el uso de tablas, y por lo tanto se hace difícil identificar rápidamente cuáles son las dimensiones más importantes, el objetivo de este trabajo es el de proponer una ecuación matemática que permita estimar el ICV directamente a partir de los valores de diferentes dimensiones, y sin requerir el uso de ninguna tabla. De esta manera, este modelo permitirá una predicción de valores del ICV en otras bases de datos, y al mismo tiempo identificará qué dimensiones tienen mayor peso en la estimación del ICV. 
Dado que la correlación entre el ICV y las diferentes dimensiones tiene una dependencia lineal, en este trabajo, hemos elegido el método de regresión múltiple lineal para obtener el modelo de regresión entre dichas variables.

En el artículo titulado Calidad de vida en ancianatos de zonas periurbanas de Asunción, Paraguay ${ }^{(16)}$, se abordaron todas las variables de estudio usando la encuesta FUMAT, de modo que el trabajo actual puede considerarse una continuación de dicho artículo.

Este trabajo se organiza de la siguiente manera. En la Sección 2, se detalla la metodología para obtener la base de datos generada y se describe la forma en que se obtiene el modelo de regresión lineal. Los resultados de la base de datos se presentan en la Sección 3. Finalmente, la regresión del ICV se presenta en la Sección 4.

\section{MATERIALES Y MÉTODOS}

\section{Metodología.}

Se realizó un estudio observacional descriptivo de corte transversal en cuatro hogares de ancianos de la zona periurbana de Asunción, Paraguay, entre los meses de febrero y marzo del año 2015. Se han recolectado datos de los hogares de ancianos: La Piedad, Nuestra Señora de la Asunción, Gigón Roga y Santo Domingo por la accesibilidad que mostraros los encargados de dichos centros permitiendo el ingreso para el relevamiento de los datos.

Participaron del estudio 33 adultos mayores de 60 años, de ambos sexos, que otorgaron su consentimiento para participar del estudio. Se excluyó del estudio a los adultos mayores que presentaban algún impedimento físico para la medición antropométrica 0 alguna alteración psicológica que dificultara contestar a las preguntas de la encuesta. Así como aquellos adultos mayores que no pudieron completar la encuesta en forma correcta y completa.

Los datos recolectados fueron consignados en una ficha individual para cada adulto mayor en el cual fueron contemplados datos sociodemográficos, de calidad de vida y actividad física.

La variable calidad de vida fue determinada a través de la aplicación de la encuesta Evaluación de la calidad de vida en adultos mayores (FUMAT), que explora las ocho dimensiones mencionadas en la Sección 1, proporcionando información confiable. El tiempo de aplicación de la encuesta fue de aproximadamente 10 minutos $^{(12)}$.

La información recopilada fue digitalizada, procesada y analizada utilizando Excel $囚$. Los datos cualitativos se expresaron en frecuencia absoluta ( $n$ ) y porcentaje (\%), y los cuantitativos en promedio y desviación estándar para variables homogéneas y medianas con los valores mínimo y máximo para aquellas de distribución no homogénea.

El protocolo de investigación fue evaluado y aprobado por el Comité de Ética de la Facultad de Ciencias Químicas de la Universidad Nacional de Asunción (Código de ética Institucional (CEI) Número 55).

La implementación de la investigación se realizó con el permiso de la autoridad máxima de cada hogar, así como de cada adulto mayor que cumplió con los criterios de selección, garantizando un trato igualitario para cada uno de los mismos. Aspectos éticos de confidencialidad, respeto, beneficencia y justicia fueron considerados en todo momento ${ }^{(17)}$.

\section{Modelo de regresión}

Para proceder a la formulación de una correlación confiable entre las distintas dimensiones y el respectivo ICV en adultos mayores, el primer paso fue la selección de un tamaño adecuado de la muestra representativa con los valores informados, que fueron descritos en la Sección 2.1. 
Nuestra intención es utilizar una muestra lo suficientemente amplia para que el modelo propuesto sea robusto. Particularmente en lo que respecta a las dimensiones elegidas para la correlación, estas fueron las anteriormente presentadas. La ecuación de regresión elegida para el análisis de regresión lineal múltiple es de la forma:

ICV $=A+a_{1} x_{1}+a_{2} x_{2}+a_{3} x_{3}+a_{4} x_{4}+a_{5} x_{5}+a_{6} x_{6}+a_{7} x_{7}+a_{8} x_{8}$

con los coeficientes $A, a_{1}-a_{8}$ a ser calculados usando el método de los mínimos cuadrados. $x_{1}$ a $x_{8}$ son los valores de las ocho dimensiones: Bienestar emocional $\left(x_{1}\right)$, Relaciones interpersonales $\left(x_{2}\right)$, Bienestar material $\left(x_{3}\right)$, Desarrollo personal $\left(x_{4}\right)$, Bienestar físico $\left(x_{5}\right)$, Autodeterminación $\left(x_{6}\right)$, Inclusión social $\left(x_{7}\right)$ y Derechos $\left(x_{8}\right)$.

La correlación obtenida se comparará luego con datos obtenidos en la base de datos, con el fin de evaluar su capacidad predictiva.

\section{RESULTADOS}

En la Tabla 1 se aprecia cada uno de los aspectos del ICV de los adultos mayores que fueron evaluados por dimensión tras la aplicación de la encuesta FUMAT (12).

Como se puede ver en la Tabla 1, dentro del bienestar material se encontró que el mayor porcentaje refirió no tener problemas de comportamiento $(88 \%, n=29)$ y que nunca o casi nunca se muestran satisfechos con los servicios que recibe $(85 \%, n=28)$. En cuanto a las relaciones interpersonales manifestaron que nunca o casi nunca tienen buena relación con los profesionales del hogar en el que se encuentran $(82 \%, n=27)$. Expresaron también que el lugar en el que se encuentran albergados no es confortable $(82 \%, n=27)$ por lo que el bienestar material es un aspecto evidentemente muy abandonado. A pesar de ello y tras evaluar el desarrollo personal de los adultos mayores, los mismos mostraron responsabilidad en la toma de sus medicamentos $(55 \%, n=18)$. Respecto al bienestar físico, la mayoría expresó no tener problemas de continencia $(85 \%, n=28)$ ni de movilidad $(64 \%, n=21)$ visualizado claramente en el elevado número de adultos mayores que a pesar del estado de salud individual por el que se encontraban pasando podían salir a la calle siempre o casi siempre $(30 \%$, $\mathrm{n}=10$ ). El mayor porcentaje de los encuestados no ha elegido el lugar en el que se encuentra actualmente $(61 \%, \mathrm{n}=21)$ y frecuentemente son otras las personas quienes toman decisiones importantes en la vida de los adultos mayores $(45 \%, n=15)$. De hecho, otro aspecto bastante desfavorecido es el de los derechos ya que nunca o casi nunca los respetan o defienden $(48 \%, n=16)$, así como también solo algunas veces reciben información adecuada y suficiente sobre los tratamientos e intervenciones que reciben $(39 \%, n=13)$.

Tabla 1. Frecuencia sobre calidad de vida según los aspectos evaluados en la encuesta FUMAT a la muestra de estudio.

\begin{tabular}{|c|c|c|c|c|c|c|c|c|}
\hline \multirow[t]{2}{*}{ Bienestar emocional } & \multicolumn{2}{|c|}{$\begin{array}{l}\text { Siempre o } \\
\text { casi siempre }\end{array}$} & \multicolumn{2}{|c|}{$\begin{array}{l}\text { Frecuen- } \\
\text { temente }\end{array}$} & \multicolumn{2}{|c|}{$\begin{array}{l}\text { Algunas } \\
\text { veces }\end{array}$} & \multicolumn{2}{|c|}{$\begin{array}{l}\text { Nunca o casi } \\
\text { nunca }\end{array}$} \\
\hline & $\mathbf{n}$ & $\%$ & $\mathbf{n}$ & $\%$ & $\mathbf{n}$ & $\%$ & $\mathbf{n}$ & $\%$ \\
\hline $\begin{array}{l}\text { 1. En general, se muestra satisfecho } \\
\text { con su vida presente }\end{array}$ & 4 & 12 & 6 & 18 & 7 & 21 & 16 & 48 \\
\hline 2.Manifiesta sentirse inútil & 6 & 18 & 5 & 15 & 9 & 27 & 13 & 39 \\
\hline 3.Se muestra intranquilo o nervioso & 6 & 18 & 3 & 9 & 6 & 18 & 18 & 55 \\
\hline $\begin{array}{l}\text { 4.Se muestra satisfecho consigo } \\
\text { mismo }\end{array}$ & 1 & 3 & 5 & 15 & 7 & 21 & 20 & 61 \\
\hline $\begin{array}{l}\text { 5.Tiene problemas de } \\
\text { comportamiento }\end{array}$ & 0 & 0 & 1 & 3 & 3 & 9 & 29 & 88 \\
\hline $\begin{array}{l}\text { 6.Se muestra satisfecho con los } \\
\text { servicios y los apoyos que recibe }\end{array}$ & 1 & 3 & 4 & 12 & 0 & 0 & 28 & 85 \\
\hline $\begin{array}{l}\text { 7. Manifiesta sentirse triste o } \\
\text { deprimido }\end{array}$ & 2 & 6 & 2 & 6 & 10 & 30 & 19 & 58 \\
\hline $\begin{array}{l}\text { 8. Muestra sentimientos de } \\
\text { incapacidad e inseguridad }\end{array}$ & 3 & 9 & 1 & 3 & 11 & 33 & 18 & 55 \\
\hline
\end{tabular}




\begin{tabular}{|c|c|c|c|c|c|c|c|c|}
\hline \multirow[t]{2}{*}{ Relaciones interpersonales } & \multicolumn{2}{|c|}{$\begin{array}{l}\text { Siempre o } \\
\text { casi siempre }\end{array}$} & \multicolumn{2}{|c|}{$\begin{array}{l}\text { Frecuente } \\
\text { mente }\end{array}$} & \multicolumn{2}{|c|}{$\begin{array}{l}\text { Algunas } \\
\text { veces }\end{array}$} & \multicolumn{2}{|c|}{$\begin{array}{l}\text { Nunca o casi } \\
\text { nunca }\end{array}$} \\
\hline & $\mathbf{n}$ & $\%$ & $\mathbf{n}$ & $\%$ & $\mathbf{n}$ & $\%$ & $\mathbf{n}$ & $\%$ \\
\hline $\begin{array}{l}\text { 9. Realiza actividades que le gustan } \\
\text { con otras personas }\end{array}$ & 5 & 15 & 9 & 27 & 5 & 15 & 14 & 42 \\
\hline $\begin{array}{l}\text { 10. Mantiene una buena relación con } \\
\text { los profesionales del servicio al que } \\
\text { acude } \\
\text { 11. Mantiene una buena relación con }\end{array}$ & 1 & 3 & 3 & 9 & 2 & 6 & 27 & 82 \\
\hline $\begin{array}{l}\text { sus compañeros del servicio al que } \\
\text { acude }\end{array}$ & 3 & 9 & 3 & 9 & 2 & 6 & 25 & 76 \\
\hline 12. Carece de familiares cercanos & 11 & 33 & 5 & 15 & 2 & 6 & 15 & 45 \\
\hline $\begin{array}{l}\text { 13.Valora negativamente sus } \\
\text { relaciones de amistad }\end{array}$ & 4 & 12 & 3 & 9 & 2 & 6 & 24 & 73 \\
\hline $\begin{array}{l}\text { 14. Manifiesta sentirse querido por las } \\
\text { personas importantes para Él/ Ella }\end{array}$ & 5 & 15 & 7 & 21 & 5 & 15 & 16 & 48 \\
\hline \multirow[t]{2}{*}{ Bienestar material } & \multicolumn{2}{|c|}{$\begin{array}{l}\text { Siempre o } \\
\text { casi siempre }\end{array}$} & \multicolumn{2}{|c|}{$\begin{array}{l}\text { Frecuente } \\
\text { mente }\end{array}$} & \multicolumn{2}{|c|}{$\begin{array}{l}\text { Algunas } \\
\text { veces }\end{array}$} & \multicolumn{2}{|c|}{$\begin{array}{l}\text { Nunca o casi } \\
\text { nunca }\end{array}$} \\
\hline & $\mathbf{n}$ & $\%$ & $\mathbf{n}$ & $\%$ & $\mathbf{n}$ & $\%$ & $\mathbf{n}$ & $\%$ \\
\hline $\begin{array}{l}\text { 15. El lugar donde vive es } \\
\text { confortable } \\
\text { 16. Manifiesta no estar satisfecho }\end{array}$ & 0 & 0 & 3 & 9 & 3 & 9 & 27 & 82 \\
\hline $\begin{array}{l}\text { con su jubilación (o situación laboral } \\
\text { actual) }\end{array}$ & 9 & 27 & 1 & 3 & 3 & 9 & 20 & 61 \\
\hline $\begin{array}{l}\text { 17. Se queja de su salario (o } \\
\text { pensión) }\end{array}$ & 10 & 30 & 1 & 3 & 1 & 3 & 21 & 64 \\
\hline $\begin{array}{l}\text { 18. El lugar donde vive tiene } \\
\text { barreras arquitectónicas que impiden } \\
\text { o dificultan alguna de sus actividades } \\
\text { 19. El servicio al que acude tiene }\end{array}$ & 0 & 0 & 2 & 6 & 11 & 33 & 20 & 61 \\
\hline $\begin{array}{l}\text { barreras arquitectónicas que impiden } \\
\text { o dificultan alguna de sus actividades }\end{array}$ & 0 & 0 & 1 & 3 & 12 & 36 & 20 & 61 \\
\hline $\begin{array}{l}\text { 20. Dispone de las cosas materiales } \\
\text { que necesita }\end{array}$ & 2 & 6 & 1 & 3 & 7 & 21 & 23 & 70 \\
\hline $\begin{array}{l}\text { 21. El lugar donde vive necesita } \\
\text { reformas para adaptarse a sus } \\
\text { necesidades }\end{array}$ & 3 & 9 & 3 & 9 & 7 & 21 & 20 & 61 \\
\hline \multirow[t]{2}{*}{ Desarrollo personal } & \multicolumn{2}{|c|}{$\begin{array}{l}\text { Siempre o } \\
\text { casi siempre }\end{array}$} & \multicolumn{2}{|c|}{$\begin{array}{l}\text { Frecuente } \\
\text { mente }\end{array}$} & \multicolumn{2}{|c|}{$\begin{array}{l}\text { Algunas } \\
\text { veces }\end{array}$} & \multicolumn{2}{|c|}{$\begin{array}{l}\text { Nunca o casi } \\
\text { nunca }\end{array}$} \\
\hline & n & $\%$ & $\mathbf{n}$ & $\%$ & $\mathbf{n}$ & $\%$ & $\mathbf{n}$ & $\%$ \\
\hline $\begin{array}{l}\text { 22. Puede leer información básica } \\
\text { para la vida cotidiana (carteles, } \\
\text { periódico, etc.) } \\
\text { 23. Muestra dificultad para resolver }\end{array}$ & 8 & 24 & 5 & 15 & 3 & 9 & 17 & 52 \\
\hline $\begin{array}{l}\text { con eficacia los problemas que se le } \\
\text { plantean }\end{array}$ & 2 & 6 & 3 & 9 & 8 & 24 & 20 & 61 \\
\hline $\begin{array}{l}\text { 24.Tiene dificultades para expresar } \\
\text { información }\end{array}$ & 0 & 0 & 1 & 3 & 9 & 27 & 23 & 70 \\
\hline $\begin{array}{l}25 . \text { En el servicio al que acude le } \\
\text { proporcionan información sobre } \\
\text { cuestiones que le interesan }\end{array}$ & 5 & 15 & 3 & 9 & 9 & 27 & 16 & 48 \\
\hline $\begin{array}{l}\text { 26. Muestra dificultades para } \\
\text { manejar conceptos matemáticos } \\
\text { básicos, útiles para la vida cotidiana } \\
\text { (sumar, restar, etc.) } \\
\text { 27.Tiene dificultades para }\end{array}$ & 4 & 12 & 0 & 0 & 8 & 24 & 21 & 64 \\
\hline $\begin{array}{l}\text { comprender la información que } \\
\text { recibe }\end{array}$ & 0 & 0 & 1 & 3 & 4 & 12 & 28 & 85 \\
\hline $\begin{array}{l}\text { 28.Es responsable de la toma de su } \\
\text { medicación }\end{array}$ & 18 & 55 & 2 & 6 & 2 & 6 & 11 & 33 \\
\hline 29.Muestra escasa flexibilidad mental & 4 & 12 & 0 & 0 & 3 & 9 & 26 & 79 \\
\hline \multirow[t]{2}{*}{ Bienestar físico } & \multicolumn{2}{|c|}{$\begin{array}{l}\text { Siempre o } \\
\text { casi siempre }\end{array}$} & \multicolumn{2}{|c|}{$\begin{array}{l}\text { Frecuente } \\
\text { mente }\end{array}$} & \multicolumn{2}{|c|}{$\begin{array}{l}\text { Algunas } \\
\text { veces }\end{array}$} & \multirow{2}{*}{\multicolumn{2}{|c|}{$\begin{array}{l}\text { Nunca o casi } \\
\text { nunca }\end{array}$}} \\
\hline & n & $\%$ & n & $\%$ & n & $\%$ & & \\
\hline \multirow{4}{*}{$\begin{array}{l}\text { 30.Tiene problemas de movilidad } \\
\text { 31.Tiene problemas de continencia } \\
\text { 32.Tiene dificultad para seguir una } \\
\text { conversación porque oye mal } \\
\text { 33.Su estado de salud le permite } \\
\text { salir a la calle }\end{array}$} & 4 & 12 & 4 & 12 & 4 & 12 & 21 & 64 \\
\hline & 3 & 9 & 1 & 3 & 1 & 3 & 28 & 85 \\
\hline & 1 & 3 & 2 & 6 & 8 & 24 & 22 & 67 \\
\hline & 10 & 30 & 9 & 27 & 9 & 27 & 5 & 15 \\
\hline
\end{tabular}


34. Tiene problemas para recordar información importante para la vida cotidiana (caras familiares, nombres, etc.)

\begin{tabular}{|c|c|c|c|c|c|c|c|c|}
\hline \multirow[t]{2}{*}{ Autodeterminación } & \multicolumn{2}{|c|}{$\begin{array}{l}\text { Siempre o } \\
\text { casi siempre }\end{array}$} & \multicolumn{2}{|c|}{$\begin{array}{l}\text { Frecuente } \\
\text { mente }\end{array}$} & \multicolumn{2}{|c|}{$\begin{array}{l}\text { Algunas } \\
\text { veces }\end{array}$} & \multicolumn{2}{|c|}{$\begin{array}{l}\text { Nunca o casi } \\
\text { nunca }\end{array}$} \\
\hline & & $\%$ & $\mathbf{n}$ & $\%$ & n & $\%$ & $\mathbf{n}$ & $\%$ \\
\hline $\begin{array}{l}\text { 35.Tiene dificultades de visión que le } \\
\text { impiden realizar sus tareas } \\
\text { habituales }\end{array}$ & 7 & 21 & 4 & 12 & 6 & 18 & 16 & 48 \\
\hline $\begin{array}{l}\text { 36. Hace planes sobre su futuro } \\
\text { 37. Muestra dificultades para }\end{array}$ & 15 & 45 & 6 & 18 & 9 & 27 & 3 & 9 \\
\hline $\begin{array}{l}\text { manejar el dinero de forma } \\
\text { autónoma (cheques, alquiler, } \\
\text { facturas, ir al banco, etc.) }\end{array}$ & 10 & 30 & 0 & 0 & 5 & 15 & 18 & 55 \\
\hline \multirow{5}{*}{$\begin{array}{l}\text { 38.Otras personas organizan su vida } \\
\text { 39. Elige cómo pasar su tiempo libre } \\
\text { 40. Ha elegido el lugar donde vive } \\
\text { actualmente } \\
\text { 41.Su familia respeta sus decisiones } \\
\text { 42. Toma decisiones sobre cuestiones } \\
\text { cotidianas }\end{array}$} & 10 & 30 & 12 & 36 & 8 & 24 & 2 & 6 \\
\hline & 2 & 6 & 8 & 24 & 11 & 33 & 12 & 36 \\
\hline & 20 & 61 & 2 & 6 & 0 & 0 & 11 & 33 \\
\hline & 16 & 48 & 5 & 15 & 6 & 18 & 6 & 18 \\
\hline & 1 & 3 & 15 & 45 & 12 & 36 & 5 & 15 \\
\hline \multirow{2}{*}{ Inclusión social } & \multicolumn{2}{|c|}{$\begin{array}{l}\text { Siempre o } \\
\text { casi siempre }\end{array}$} & \multicolumn{2}{|c|}{$\begin{array}{l}\text { Frecuente } \\
\text { mente }\end{array}$} & \multicolumn{2}{|c|}{$\begin{array}{l}\text { Algunas } \\
\text { veces }\end{array}$} & \multicolumn{2}{|c|}{$\begin{array}{l}\text { Nunca o casi } \\
\text { nunca }\end{array}$} \\
\hline & $\mathbf{n}$ & $\%$ & $\mathbf{n}$ & $\%$ & $\mathbf{n}$ & $\%$ & $\mathbf{n}$ & $\%$ \\
\hline $\begin{array}{l}\text { 43. Otras personas toman las } \\
\text { decisiones que son importantes para } \\
\text { su vida }\end{array}$ & 6 & 18 & 15 & 45 & 9 & 27 & 3 & 9 \\
\hline $\begin{array}{l}\text { 44.Participa en diversas actividades } \\
\text { de ocio que le interesan }\end{array}$ & 4 & 12 & 9 & 27 & 7 & 21 & 13 & 39 \\
\hline $\begin{array}{l}\text { 45. Está excluido en su comunidad } \\
\text { 46. En el servicio al que acude, tiene }\end{array}$ & 4 & 12 & 2 & 6 & 12 & 36 & 15 & 45 \\
\hline $\begin{array}{l}\text { dificultad para encontrar apoyos } \\
\text { cuando los necesita }\end{array}$ & 3 & 9 & 3 & 9 & 4 & 12 & 23 & 70 \\
\hline $\begin{array}{l}\text { 47.Tiene amigos que le apoyan } \\
\text { cuando lo necesita }\end{array}$ & 11 & 33 & 5 & 15 & 6 & 18 & 11 & 33 \\
\hline \multirow{5}{*}{$\begin{array}{l}\text { 48.Tiene dificultades para } \\
\text { relacionarse con otras personas del } \\
\text { centro al que acude } \\
49 \text {. Está integrado con los } \\
\text { compañeros del servicio al que acude } \\
50 . \text { Participa de forma voluntaria en } \\
\text { algún programa o actividad del } \\
\text { servicio al que acude } \\
51 . \text { Su red de apoyos no satisface sus } \\
\text { necesidades }\end{array}$} & & & & & & & & \\
\hline & 1 & 3 & 2 & 6 & 9 & 27 & 21 & 64 \\
\hline & 2 & 6 & 6 & 18 & 6 & 18 & 19 & 58 \\
\hline & 2 & 6 & 5 & 15 & 5 & 15 & 21 & 64 \\
\hline & 4 & 12 & 3 & 9 & 4 & 12 & 22 & 67 \\
\hline \multirow[t]{2}{*}{ Derechos } & \multicolumn{2}{|c|}{$\begin{array}{l}\text { Siempre o } \\
\text { casi siempre }\end{array}$} & \multicolumn{2}{|c|}{$\begin{array}{l}\text { Frecuente } \\
\text { mente }\end{array}$} & \multicolumn{2}{|c|}{$\begin{array}{l}\text { Algunas } \\
\text { veces }\end{array}$} & \multicolumn{2}{|c|}{$\begin{array}{l}\text { Nunca o casi } \\
\text { nunca }\end{array}$} \\
\hline & n & $\%$ & $\mathbf{n}$ & $\%$ & $\mathbf{n}$ & $\%$ & $\mathbf{n}$ & $\%$ \\
\hline $\begin{array}{l}\text { 52.Tiene dificultades para participar } \\
\text { en su comunidad }\end{array}$ & 1 & 3 & 4 & 12 & 8 & 24 & 20 & 61 \\
\hline $\begin{array}{l}\text { 53.En el servicio al que acude se } \\
\text { respetan y defienden sus derechos }\end{array}$ & 2 & 6 & 7 & 21 & 8 & 24 & 16 & 48 \\
\hline $\begin{array}{l}\text { 54. Recibe información adecuada y } \\
\text { suficiente sobre los tratamientos e } \\
\text { intervenciones que recibe } \\
55 . \text { Muestra dificultad para defender }\end{array}$ & 3 & 9 & 5 & 15 & 13 & 39 & 12 & 36 \\
\hline $\begin{array}{l}\text { sus derechos cuando no son } \\
\text { respetados }\end{array}$ & 2 & 6 & 6 & 18 & 6 & 18 & 19 & 58 \\
\hline $\begin{array}{l}\text { 56. Cuenta con asistencia legal y/o } \\
\text { acceso a servicios de asesoría legal } \\
57 \text {. Disfruta de todos sus derechos }\end{array}$ & 23 & 70 & 4 & 12 & 4 & 12 & 2 & 6 \\
\hline $\begin{array}{l}\text { legales (ciudadanía, voto, procesos } \\
\text { legales, etc.) }\end{array}$ & 21 & 64 & 4 & 12 & 7 & 21 & 1 & 3 \\
\hline
\end{tabular}

Al evaluar el promedio general y la desviación estándar del ICV tras la evaluación de todos los indicadores, se encontró que corresponde a $58 \pm 23$ (valores mínimo y máximo de 14 y 93, respectivamente), encontrándose de esta forma una gran dispersión en los valores del ICV en los 33 adultos mayores (ver Tabla 2). 
Tabla 2. Promedio y desviaciones estándar (DE) de las ocho dimensiones y del ICV de los 33 adultos usando la encuesta FUMAT

\begin{tabular}{lcc}
\hline Escala de vida & Promedio $\mathbf{D E}$ & Mínimo- Máximo \\
\hline Desarrollo personal & $73 \pm 18$ & $25-95$ \\
Inclusión social & $69 \pm 24$ & $9-95$ \\
Bienestar emocional & $66 \pm 20$ & $25-98$ \\
Bienestar físico & $63 \pm 23$ & $2-91$ \\
Relaciones interpersonales & $56 \pm 25$ & $1-84$ \\
Autodeterminación & $48 \pm 24$ & $16-91$ \\
Bienestar material & $45 \pm 30$ & $0-84$ \\
Derechos & $43 \pm 19$ & $\mathbf{2 - 8 4}$ \\
\hline ICV & $\mathbf{5 8 \pm 2 3}$ & $\mathbf{1 4 - 9 3}$ \\
\hline
\end{tabular}

Por último, la Tabla 3 presenta de forma resumida y concisa los valores obtenidos en las ocho dimensiones de estudio, así como el ICV estimado usando tablas, de los 33 adultos mayores encuestados. Dicha tabla muestra una gran dispersión en los valores del ICV en los 33 adultos, indicando la utilidad de una ecuación matemática en la predicción de dichos valores con gran precisión.

Tabla 3. Valores de las ocho dimensiones e ICV de los 33 adultos mayores

\begin{tabular}{|c|c|c|c|c|c|c|c|c|c|}
\hline Adulto & $x 1$ & $\times 2$ & $\times 3$ & $x 4$ & $\times 5$ & $\times 6$ & $\times 7$ & $\times 8$ & ICV \\
\hline 1 & 13 & 13 & 12 & 13 & 11 & 13 & 15 & 11 & 117 \\
\hline 2 & 11 & 12 & 12 & 11 & 10 & 12 & 14 & 10 & 110 \\
\hline 3 & 13 & 12 & 13 & 13 & 11 & 12 & 14 & 9 & 115 \\
\hline 4 & 12 & 13 & 12 & 12 & 14 & 13 & 13 & 10 & 116 \\
\hline 5 & 12 & 9 & 10 & 12 & 11 & 13 & 11 & 10 & 106 \\
\hline 6 & 14 & 13 & 12 & 15 & 14 & 14 & 15 & 11 & 122 \\
\hline 7 & 12 & 13 & 12 & 13 & 13 & 10 & 12 & 10 & 112 \\
\hline 8 & 13 & 12 & 12 & 13 & 12 & 10 & 14 & 10 & 113 \\
\hline 9 & 12 & 8 & 7 & 9 & 4 & 9 & 12 & 6 & 90 \\
\hline 10 & 13 & 8 & 9 & 12 & 11 & 8 & 8 & 8 & 98 \\
\hline 11 & 10 & 9 & 7 & 11 & 12 & 7 & 6 & 4 & 89 \\
\hline 12 & 12 & 10 & 3 & 13 & 12 & 9 & 12 & 9 & 100 \\
\hline 13 & 10 & 12 & 10 & 11 & 11 & 10 & 12 & 9 & 104 \\
\hline 14 & 11 & 9 & 9 & 8 & 11 & 7 & 10 & 8 & 94 \\
\hline 15 & 9 & 12 & 2 & 13 & 11 & 10 & 9 & 9 & 96 \\
\hline 16 & 10 & 11 & 4 & 15 & 12 & 7 & 10 & 8 & 98 \\
\hline 17 & 12 & 11 & 10 & 13 & 11 & 12 & 14 & 13 & 113 \\
\hline 18 & 9 & 4 & 6 & 11 & 9 & 7 & 9 & 6 & 85 \\
\hline 19 & 9 & 13 & 10 & 11 & 8 & 10 & 13 & 11 & 104 \\
\hline 20 & 11 & 9 & 4 & 9 & 13 & 8 & 11 & 10 & 96 \\
\hline 21 & 9 & 8 & 5 & 13 & 11 & 9 & 9 & 8 & 94 \\
\hline 22 & 12 & 10 & 10 & 11 & 13 & 7 & 11 & 10 & 103 \\
\hline 23 & 9 & 9 & 8 & 13 & 11 & 12 & 14 & 8 & 103 \\
\hline 24 & 10 & 6 & 12 & 11 & 9 & 9 & 10 & 8 & 96 \\
\hline 25 & 12 & 11 & 12 & 9 & 13 & 9 & 13 & 9 & 106 \\
\hline 26 & 13 & 8 & 4 & 11 & 9 & 10 & 11 & 10 & 97 \\
\hline 27 & 15 & 9 & 5 & 14 & 13 & 9 & 11 & 10 & 105 \\
\hline 28 & 9 & 13 & 2 & 13 & 5 & 10 & 14 & 10 & 97 \\
\hline 29 & 12 & 13 & 11 & 12 & 11 & 13 & 13 & 11 & 113 \\
\hline 30 & 13 & 12 & 10 & 13 & 12 & 7 & 13 & 10 & 108 \\
\hline 31 & 8 & 8 & 1 & 11 & 9 & 10 & 8 & 8 & 86 \\
\hline 32 & 14 & 12 & 12 & 15 & 13 & 11 & 15 & 12 & 119 \\
\hline 33 & 11 & 12 & 12 & 15 & 13 & 9 & 12 & 11 & 112 \\
\hline
\end{tabular}

En la Figura 1 representamos en la coordenada de las abscisas los valores de ICV presentados en la Tabla 3, en el rango disponible de datos. En el eje de ordenadas 
representamos los valores de ICV estimados por la regresión propuesta en este trabajo. También se muestra una línea roja con pendiente 1 (recta a $45^{\circ}$ ). Cuando los valores obtenidos a través de la regresión se encuentran en esta recta, una concordancia perfecta entre el valor de la Tabla 3 y el valor obtenido con la regresión se puede observar. Cuando yacen lejos, la precisión de la ecuación de regresión es bastante pobre para aproximarse a los datos iniciales.

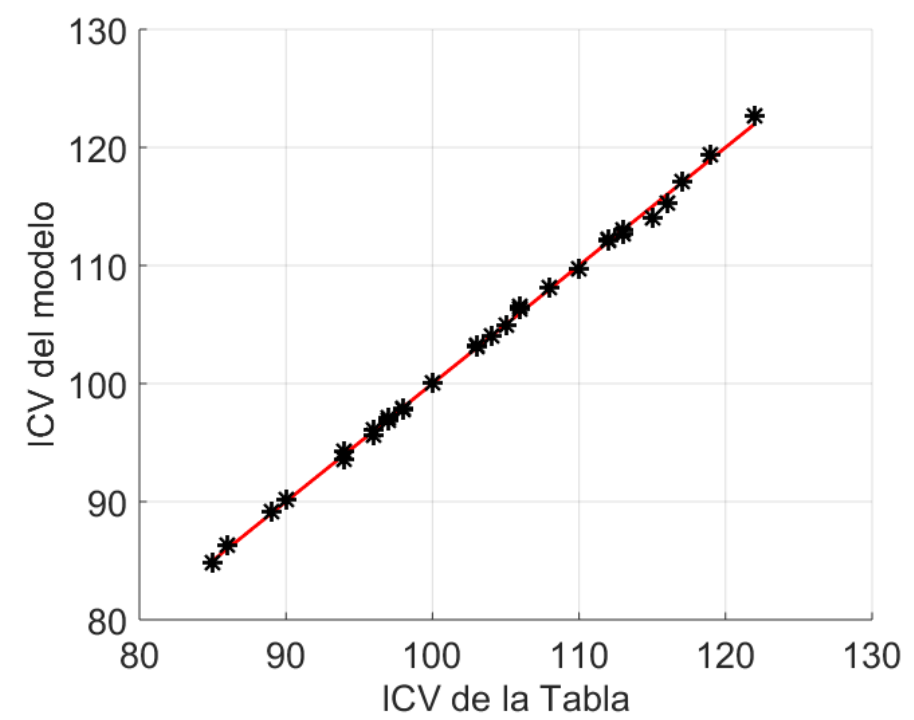

Figura 1. Predicción del ICV usando la ecuación propuesta, en función a los datos presentados en la Tabla 3.

\section{DISCUSIón}

Tras la aplicación de la encuesta FUMAT, se encontró que el promedio más bajo con respecto a los indicadores, es decir, los más desfavorecidos corresponden a los aspectos relacionados al derecho, el bienestar material y la autodeterminación de los adultos (ver Tabla 2). Mientras que dentro de la escala de calidad de vida, el promedio más alto fue para desarrollo personal. Estos hallazgos contrastan con el estudio de Quintanar Guzmán (18), realizado en una población adulta mayor institucionalizada del estado de Hidalgo en el 2010, Colombia, que concluyó que los aspectos concernientes a la inclusión social y relaciones interpersonales son los más afectados. Sin embargo, cabe resaltar que tanto en este estudio como en el mencionado se encontró un porcentaje importante de insatisfacción o de calidad de vida baja relacionado al bienestar material, que está relacionado al lugar donde viven, a la jubilación, entre otros. Las diferencias entre ambas observaciones podrían deberse a que la investigación de Quintanar Guzmán fue realizada con voluntarios que se trasladaron al lugar de toma de datos mientras que la presente investigación fue hecha en cada hogar. Asimismo, el WHOQOL-BREF ${ }^{(19-21)}$, cuestionario utilizado por Quintanar, es un instrumento de 26 ítems mientras que el FUMAT evalúa 57 preguntas. Asimismo, los datos de esta observación no coinciden con lo reportado por Ramírez et al. ${ }^{(22)}$, en el que se distinguen las puntuaciones más bajas para las dimensiones de rol emocional y la función social. Cabe destacar que en la investigación de Ramírez se empleó la escala de Cuestionario de Salud General (SF12), que resulta de una versión resumida de otro instrumento. Asimismo, ésta es la ideal para la evaluación pretratamiento, evaluación durante el tratamiento, y evaluación post-tratamiento. La escala FUMAT es un instrumento para evaluar la calidad de vida objetiva de personas mayores que son usuarias de servicios sociales o institucionalizados, por lo que los resultados son diferentes debido a que los instrumentos empleados no son los mismos ${ }^{(12-13)}$.

Como se describe en la Sección 2.2, una regresión múltiple lineal se implementó en este trabajo. El mejor acuerdo (menor error cuadrático medio, o RMSE por sus siglas en inglés) con respecto a la base de datos presentada en la Tabla 3, se encontró usando la ecuación ${ }^{(2)}$. Dicha ecuación se compone de un término constante, y 
términos lineales para cada dimensión. Cabe señalar que otras técnicas de regresión consideran ecuaciones con términos cuadráticos, cúbicos o de orden superior, e incluso términos no lineales para todas las variables independientes (dimensiones). Sin embargo, estos modelos fueron descartados ya que el modelo de regresión lineal muestra un buen acuerdo con la base de datos, lo que sugiere que el ICV se puede estimar correctamente usando esta sencilla ecuación.

Aplicando el análisis de regresión lineal múltiple con el método de los mínimos cuadrados, la ecuación ${ }^{(1)}$ se transforma en:

ICV $=35.71+0.86 x_{1}+0.84 x_{2}+0.81 x_{3}+0.85 x_{4}+0.75 x_{5}+0.82 x_{6}+0.78 x_{7}+0.71 x_{8}$

El primer término (constante) en la ecuación (2) es relativamente bajo, teniendo en cuenta el rango de valores del ICV de la Tabla 3, por lo que el peso de los restantes 8 términos dependientes de las distintas dimensiones es bastante importante. Los signos positivos de $a_{1}$ a $a_{8}$ indican que el ICV aumenta al aumentar los valores de las distintas dimensiones. Por lo tanto, la ecuación matemática es coherente con la dependencia de las distintas dimensiones y el valor estimado del ICV. Por lo que además de tener un buen acuerdo matemático, reproduce la dependencia del ICV con las distintas dimensiones. Los valores de $\mathrm{a}_{1} \mathrm{a} \mathrm{a}_{8}$ son del mismo orden de grandeza. Esto indica que el peso de cada dimensión es aproximadamente el mismo en la estimación del ICV. Sin embargo, valores ligeramente inferiores se encuentran para las dimensiones $\mathrm{x}_{8}$ (Derechos) y $\mathrm{x}_{5}$ (Bienestar físico), indicando una dependencia ligeramente menos fuerte para estas dimensiones, en relación al resto.

La Figura 1 muestra que los valores predichos por la ecuación de regresión siguen la tendencia de los datos iniciales. Los coeficientes estadísticos del modelo usando los 33 datos son muy buenos (valores de error estándar y RMSE de $0.22 \%$ y 0.10 , respectivamente), ya que los valores predichos son consistentes con las dependencias de ICV con las distintas dimensiones.

En conclusión, el modelo de regresión del ICV obtenido a partir de los valores de las distintas dimensiones muestra una muy buena correlación con los datos iniciales, lo que demuestra la capacidad de predicción de la ecuación propuesta.

Para futuros análisis, se podrá establecer la dependencia del ICV con términos cruzados de las distintas dimensiones, de forma tal a evaluar la posibilidad de incluir dichos términos en la ecuación de regresión.

\section{AGRADECIMIENTOS}

Los autores quisieran agradecer a la Facultad de Medicina de la Universidad María Auxiliadora por el apoyo brindado para la realización de esta investigación.

\section{Conflicto de interés}

Los autores declaran que no hay ningún conflicto de interés.

\section{Contribución de los autores}

Todos los autores han contribuido en la elaboración del presente manuscrito.

\section{REFERENCIAS BIBLIÓGRAFICAS}

1. López-Rincón $F$ J, Morales-Jinez $A$, Ugarte-Esquivel A, Rodríguez-Mejía L E, Hernández-Torres J L, Sauza-Niño L C. Comparación de la percepción de calidad de vida relacionada con la salud en hombres y mujeres adultos mayores. Enfermería global 2019, 18(54), 410-425.

2. Soria Romero Z, Montoya Arce B J. Envejecimiento y factores asociados a la calidad de vida de los adultos mayores en el Estado de México. Papeles de población 2017, 23(93), 59-93.
3. Olivi A, Fadda Cori G, Pizzi Kirschbaum M. Evaluación de la calidad de vida de los adultos mayores en la ciudad de Valparaíso. Papeles de población 2015, 21(84), 227-249.

4. Mora M, Villalobos D, Araya G, Ozols A. Perspectiva subjetiva de la calidad de vida del adulto mayor, diferencias ligadas al género y a la práctica de la actividad físico recreativa. Revista MHSalud 2004; 1(1):1-12.

5. Guzmán-Muñoz $E$, Concha-Cisternas $Y$, Oñate-Barahona A, Lira-Cea C, 
Cigarroa-Cuevas I, Méndez-Rebolledo $\mathrm{G}$, et al. Factores asociados a una baja calidad de vida en adultos chilenos durante la cuarentena por COVID19. Revista médica de Chile 2020, 148(12), 1759-1766.

6. Herrera $E M$, López $A$ A, Pérez $B C$. Factores asociados a la calidad de vida de adultos mayores. Cartagena (Colombia). Salud Uninorte 2012, 28(2), 251-263.

7. Vargas-Ricardo $S \mathrm{R}$, Melguizo-Herrera E. Calidad de vida en adultos mayores en Cartagena, Colombia. Revista de Salud Pública 2017, 19, 549-554.

8. Corugedo Rodríguez M D C, García González D, González Arias V I, Crespo Lechuga G A, González García G, Calderín Hernández M. Calidad de vida en adultos mayores del hogar de ancianos del municipio Cruces. Revista Cubana de Medicina General Integral 2014, 30(2).

9. Cardona-Arias J A, Álvarez-Mendieta M I, Pastrana-Restrepo S. Calidad de vida relacionada con la salud en adultos mayores de hogares geriátricos, Medellín, Colombia, 2012. Revista Ciencias de la Salud 2014, 12(2), 139155.

10. Tuesca Molina R. La Calidad de Vida, su importancia y cómo medirla. Salud Uninorte 2005; 21:76-86.

11. Martínez MA, Leiva AM, Petermann $F$, Garrido A, Diaz X, Alvarez C, et al. Correlates of sedentary behaviors in Chile: evidence from the National Health Survey 2009-2010. Revista médica de Chile 2018, 146(1), 22-31.

12. Verdugo Alonso M, Gómez Sánchez L, Arias Martínez B. Evaluación de la calidad de vida en personas mayores: La escala FUMAT. Salamanca: Instituto Universitario de Integración en la Comunidad; 2009.

13. Gómez $L$, Verdugo $M$, Arias $B$, Navas $P$. Evaluación de la calidad de vida en personas mayores y con discapacidad: la Escala FUMAT. Intervención Psicosocial 2008.17 (2): 189-99.

14. Guede Rojas F, Chirosa Ríos L J, Fuentealba Urra S, Vergara Ríos C, Ulloa Díaz D, Campos Jara C, et al. Asociación predictiva entre parámetros de condición física y dimensiones de calidad de vida relacionada con la salud en adultos mayores chilenos insertos en la comunidad. Revista médica de Chile 2017, 145(1), 55-62.
15. Alviso D, Romano S D. Prediction of the refractive index and speed of sound of biodiesel from its composition and molecular structure. Fuel 2021 (304): 120606.

16. Meza-Paredes J, Aguilar-Rabito A, Díaz A. Calidad de vida en ancianatos de zonas periurbanas de Asunción, Paraguay. Rev. Soc. cient. Parag 2020; 25(1):6-19.

17. Asociación Médica Mundial. Declaración de Helsinki de la Asociación Médica Mundial: principios éticos para las investigaciones médicas en seres humanos Adoptada por la $18^{a}$ Asamblea Médica Mundial, Helsinki, Finlandia, Junio 1964 y enmendada por la 29a Asamblea Médica Mundial, Tokio, Japón, Octubre 1975, 35a Asamblea Médica Mundial, Venecia, Italia, Octubre 1983, $41^{a}$ Asamblea Médica Mundial, Hong Kong, Septiembre 1989, 48a Asamblea General, Somerset West, Sudáfrica, Octubre1996 y la 52a Asamblea General, Edimburgo, Escocia, Octubre 2000. 59a Asamblea General, Seúl, Corea, octubre 2008.

18. Quintanar Guzmán A. Análisis de la calidad de vida en adultos mayores del municipio de Tetepango, Hidalgo: a través del instrumento WHOQOL-Bref (tesina). Hidalgo: Escuela Superior Actopan, Área académica de psicología, Universidad Autónoma del Estado de Hidalgo; 2010.

19. Espinoza I, Osorio P, Torrejón M J, Lucas-Carrasco R, Bunout D. Validación del cuestionario de calidad de vida (WHOQOL-BREF) en adultos mayores chilenos. Revista médica de Chile 2011, vol. 139, no 5, p. 579-586.

20. Torres M, Quezada M, Rioseco R, Ducci $M$ E. Calidad de vida de adultos mayores pobres de viviendas básicas: Estudio comparativo mediante uso de WHOQOL-BREF. Revista médica de Chile 2008, vol. 136, no 3, p. 325-333.

21. Urzúa A, Navarrete M. Calidad de vida en adultos mayores: análisis factoriales de las versiones abreviadas del WHOQoL-Old en población chilena. Revista médica de Chile 2013, 141(1), 28-33.

22. Ramírez R, Agrego R, Jerez A, Chapal L. Calidad de Vida y Condiciones de Salud en Adultos Mayores no Institucionalizados en Cali, Colombia. Rev. salud pública 2008. 10 (4):529-36. 